\title{
Global maximal inequality to a class of oscillatory integrals
}

Ying Xue ${ }^{1}$ and Yaoming $\mathrm{Niu}^{1 *}$

\author{
"Correspondence: \\ nymmath@126.com \\ 'Faculty of Mathematics, Baotou \\ Teachers' College of Inner Mongolia \\ University of Science and \\ Technology, Baotou, P.R. China
}

\author{
Abstract \\ In the present paper, we give the global $L^{9}$ estimates for maximal operators \\ generated by multiparameter oscillatory integral $S_{t, \Phi}$, which is defined by

$$
S_{t, \Phi} f(x)=(2 \pi)^{-n} \int_{\mathbb{R}^{n}} e^{i x \cdot \xi} e^{i\left(t_{1} \phi_{1}\left(\left|\xi_{1}\right|\right)+t_{2} \phi_{2}\left(\left|\xi_{2}\right|\right)+\cdots+t_{n} \phi_{n}\left(\left|\xi_{n}\right|\right)\right)} \hat{f}(\xi) d \xi, \quad x \in \mathbb{R}^{n}
$$ \\ where $n \geq 2$ and $f$ is a Schwartz function in $\mathcal{S}\left(\mathbb{R}^{n}\right), t=\left(t_{1}, t_{2}, \ldots, t_{n}\right)$, \\ $\Phi=\left(\phi_{1}, \phi_{2}, \ldots, \phi_{n}\right), \phi_{i}(i=1,2,3, \ldots, n)$ is a function on $\mathbb{R}^{+} \rightarrow \mathbb{R}$, which has a suitable \\ growth condition. These estimates are apparently good extensions to the results of \\ Sjölin and Soria (J. Math. Anal. Appl 411:129-143, 2014) for the multiparameter \\ fractional Schrödinger equation.
}

MSC: $42 \mathrm{~B} 15 ; 42 \mathrm{~B} 25$

Keywords: Maximal operator; Local estimate; Global estimate; Multiparameter oscillatory integrals

\section{Introduction and main results}

Let $f$ be a Schwartz function in $\mathcal{S}\left(\mathbb{R}^{n}\right)$ and

$$
S_{t} f(x)=u(x, t)=(2 \pi)^{-n} \int_{\mathbb{R}^{n}} e^{i x \cdot \xi+i t|\xi|^{a}} \hat{f}(\xi) d \xi, \quad(x, t) \in \mathbb{R}^{n} \times \mathbb{R} .
$$

It is well known that $S_{t} f(x)$ is the solution of the fractional Schrödinger equation

$$
\left\{\begin{array}{l}
i \partial_{t} u+(-\Delta)^{a / 2} u=0, \quad(x, t) \in \mathbb{R}^{n} \times \mathbb{R} \\
u(x, 0)=f(x)
\end{array}\right.
$$

Here $\hat{f}$ denotes the Fourier transform of $f$ defined by $\hat{f}(\xi)=\int_{\mathbb{R}^{n}} e^{-i \xi \cdot x} f(x) d x$.

We recall the homogeneous Sobolev space $\dot{H}^{s}\left(\mathbb{R}^{n}\right)(s \in \mathbb{R})$, which is defined by

$$
\dot{H}^{s}\left(\mathbb{R}^{n}\right)=\left\{f \in \mathcal{S}^{\prime}:\|f\|_{H^{s}}=\left(\int_{\mathbb{R}^{n}}|\xi|^{2 s}|\hat{f}(\xi)|^{2} d \xi\right)^{1 / 2}<\infty\right\},
$$

\section{Springer}

(c) The Author(s) 2018. This article is distributed under the terms of the Creative Commons Attribution 4.0 International License (http://creativecommons.org/licenses/by/4.0/), which permits unrestricted use, distribution, and reproduction in any medium, provided you give appropriate credit to the original author(s) and the source, provide a link to the Creative Commons license, and indicate if changes were made. 
and the non-homogeneous Sobolev space $H^{s}\left(\mathbb{R}^{n}\right)(s \in \mathbb{R})$, which is defined by

$$
H^{s}\left(\mathbb{R}^{n}\right)=\left\{f \in \mathcal{S}^{\prime}:\|f\|_{H^{s}}=\left(\int_{\mathbb{R}^{n}}\left(1+|\xi|^{2}\right)^{s}|\hat{f}(\xi)|^{2} d \xi\right)^{1 / 2}<\infty\right\}
$$

Maximal operator $S^{*} f$ associated with the family of operators $\left\{S_{t}\right\}_{0<t<1}$ is defined by

$$
S^{*} f(x)=\sup _{0<t<1}\left|S_{t} f(x)\right|, \quad x \in \mathbb{R}^{n} .
$$

It is well known that if $a=2, u$ is the solution of the Schrödinger equation

$$
\left\{\begin{array}{l}
i \partial_{t} u-\Delta u=0, \quad(x, t) \in \mathbb{R}^{n} \times \mathbb{R}, \\
u(x, 0)=f(x) .
\end{array}\right.
$$

In 1979, Carleson [4] proposed a problem: if $f \in H^{s}\left(\mathbb{R}^{n}\right)$ for which $s$ does

$$
\lim _{t \rightarrow 0} u(x, t)=f(x), \quad \text { a.e. } x \in \mathbb{R}^{n} .
$$

Carleson first considered this problem for dimension $n=1$ in [4] and showed that the convergence (1.3) holds for $f \in H^{s}(\mathbb{R})$ with $s \geq \frac{1}{4}$, which is sharp was shown by Dahlberg and Kenig [8]. The higher dimensional case of convergence (1.3) has been studied by several authors, see [1, 2, 9, 11-13, 24, 25, 30, 34, 35] for example. In fact, by a standard argument, for $f \in H^{s}\left(\mathbb{R}^{n}\right)$, the pointwise convergence (1.3) follows from the local estimate

$$
\left\|S^{*} f\right\|_{L^{q\left(\mathbb{B}^{n}\right)}} \leq C\|f\|_{H^{s}\left(\mathbb{R}^{n}\right)}, \quad f \in H^{s}\left(\mathbb{R}^{n}\right),
$$

for some $q \geq 1$ and $s \in \mathbb{R}$. Here $\mathbb{B}^{n}$ is the unit ball centered at the origin in $\mathbb{R}^{n}$. On the other hand, the global estimates are of independent interest since they reveal global regularity properties of the corresponding oscillatory integrals. Next, we recall the global estimate

$$
\left\|S^{*} f\right\|_{L^{q\left(\mathbb{R}^{n}\right)}} \leq C\|f\|_{H^{s}\left(\mathbb{R}^{n}\right)}
$$

Estimate (1.5) and related questions have been well studied in literature, see, e.g., Carbery [3], Cowling [7], Kenig and Ruiz [21], Kenig, Ponce, and Vega [20], Rogers and Villarroya [29], Rogers [28], Sjölin [30-32], and so on.

For $n \geq 2$ and a multiindex $a=\left(a_{1}, a_{2}, \ldots, a_{n}\right)$, with $a_{j}>1$ and $f$ being a Schwartz function in $\mathcal{S}\left(\mathbb{R}^{n}\right)$, we set

$$
S_{t} f(x)=(2 \pi)^{-n} \int_{\mathbb{R}^{n}} e^{i x \cdot \xi} e^{i\left(t_{1}\left|\xi_{1}\right|^{a_{1}}+t_{2}\left|\xi_{2}\right|^{a_{2}}+\cdots+t_{n}\left|\xi_{n}\right|^{a_{n}}\right)} \hat{f}(\xi) d \xi, \quad x \in \mathbb{R}^{n},
$$

where $t=\left(t_{1}, t_{2}, \ldots, t_{n}\right) \in \mathbb{R}^{n}$. For $n \geq 2$, the local maximal operator $M^{*}$ is defined by

$$
M^{*} f(x)=\sup _{0<t_{i}<1}\left|S_{t} f(x)\right|, \quad x \in \mathbb{R}^{n},
$$

and the global maximal operator $M^{* *}$ is defined by

$$
M^{* *} f(x)=\sup _{t_{i} \in \mathbb{R}}\left|S_{t} f(x)\right|, \quad x \in \mathbb{R}^{n} .
$$


The global estimate

$$
\left\|M^{* *} f\right\|_{L^{q\left(\mathbb{R}^{n}\right)}} \leq C\|f\|_{\dot{H}^{s}\left(\mathbb{R}^{n}\right)}
$$

and

$$
\left\|M^{*} f\right\|_{L^{q}\left(\mathbb{R}^{n)}\right.} \leq C\|f\|_{H^{s}\left(\mathbb{R}^{n}\right)} .
$$

In 2014, Sjolin and Soria [32] obtained the following results.

Theorem A ([32]) Assume $n \geq 2$. Then, for every a, inequality (1.6) holds if and only if $4 \leq q<\infty$ and $s=n\left(\frac{1}{2}-\frac{1}{q}\right)$.

Theorem B ([32]) Assume $n \geq 2$. Then, for every $a$ and for $2<q<4$, inequality (1.7) holds if and only if $s \geq \frac{n}{2}-\frac{|a|}{4}+\frac{|a|}{q}-\frac{n}{q}$.

Multiparameter singular integrals and related operators have been well studied and raised considerable attention in harmonic analysis, which can been seen in the work of Stein and Fefferman in [14-17], and so on. In the present paper, we consider the maximal estimates associated with multiparameter oscillatory integral $S_{t, \Phi}$ defined by

$$
S_{t, \Phi} f(x)=(2 \pi)^{-n} \int_{\mathbb{R}^{n}} e^{i x \cdot \xi} e^{i\left(t_{1} \phi_{1}\left(\left|\xi_{1}\right|\right)+t_{2} \phi_{2}\left(\left|\xi_{2}\right|\right)+\cdots+t_{n} \phi_{n}\left(\left|\xi_{n}\right|\right)\right)} \hat{f}(\xi) d \xi, \quad x \in \mathbb{R}^{n} .
$$

Here, $n \geq 2$ and $f$ is a Schwartz function in $\mathcal{S}\left(\mathbb{R}^{n}\right), \Phi=\left(\phi_{1}, \phi_{2}, \ldots, \phi_{n}\right), \phi_{i}(i=1,2,3, \ldots, n)$ is a function on $\mathbb{R}^{+} \rightarrow \mathbb{R}$. For $n \geq 2$, the local maximal operator $M_{\Phi}^{*}$ is defined by

$$
M_{\Phi}^{*} f(x)=\sup _{0<t_{i}<1}\left|S_{t, \Phi} f(x)\right|, \quad x \in \mathbb{R}^{n}
$$

and the global maximal operator $M_{\Phi}^{* *}$ is defined by

$$
M_{\Phi}^{* *} f(x)=\sup _{t_{i} \in \mathbb{R}}\left|S_{t, \Phi} f(x)\right|, \quad x \in \mathbb{R}^{n} .
$$

The global estimates of maximal operators $M_{\Phi}^{*}$ and $M_{\Phi}^{* *}$ are defined by

$$
\left\|M_{\Phi}^{* *} f\right\|_{L^{q}\left(\mathbb{R}^{n)}\right.} \leq C\|f\|_{\dot{H}^{s}\left(\mathbb{R}^{n}\right)}
$$

and

$$
\left\|M_{\Phi}^{*} f\right\|_{L^{q}\left(\mathbb{R}^{n}\right)} \leq C\|f\|_{H^{s}\left(\mathbb{R}^{n}\right)}
$$

Assume that $\phi: \mathbb{R}^{+} \rightarrow \mathbb{R}$ satisfies:

(H1) There exists $m_{1}>1$ such that $\left|\phi^{\prime}(r)\right| \sim r^{m_{1}-1}$ and $\left|\phi^{\prime \prime}(r)\right| \gtrsim r^{m_{1}-2}$ for all $0<r<1$;

(H2) There exists $m_{2}>1$ such that $\left|\phi^{\prime}(r)\right| \sim r^{m_{2}-1}$ and $\left|\phi^{\prime \prime}(r)\right| \gtrsim r^{m_{2}-2}$ for all $r \geq 1$;

(H3) Either $\phi^{\prime \prime}(r)>0$ or $\phi^{\prime \prime}(r)<0$ for all $r>0$.

Now we state our main results as follows. 
Theorem 1.1 Assume that $n \geq 2$ and $\phi_{i}(i=1,2,3, \ldots, n)$ satisfies (H1)-(H3). If $4 \leq q<\infty$ and $s=n\left(\frac{1}{2}-\frac{1}{q}\right)$, then the global estimate (1.8) holds.

Theorem 1.2 Let $m=\left(m_{1,2}, m_{2,2}, \ldots, m_{n, 2}\right)$ and set $|m|=m_{1,2}+m_{2,2}+\cdots+m_{n, 2}$. Assume that $n \geq 2$ and $\phi_{i}(i=1,2,3, \ldots, n)$ satisfies $(\mathrm{H} 1)-(\mathrm{H} 3)$ with $m_{i, 1}>1, m_{i, 2}>1$. Then, for every $m$, inequality (1.9) holds if $2<q<4$ and $s \geq \frac{n}{2}-\frac{|m|}{4}+\frac{|m|}{q}-\frac{n}{q}$.

Remark 1.1 There are many elements $\phi$ satisfying conditions (H1)-(H3), for instance, the fractional Schrödinger equation $\left(\phi(r)=r^{a}\right)$, or $\left(\phi(r)=\left(1+r^{2}\right)^{\frac{a}{2}}\right),(a \geq 1)$, the Beam equation $\left(\phi(r)=\sqrt{1+r^{4}}\right)$, the fourth-order Schrödinger equation $\left(\phi(r)=r^{2}+r^{4}\right)$, iBq $\left(\phi(r)=r \sqrt{1+r^{2}}\right)$, and so on (see [5, 6, 18, 19, 22, 23, 27], and the references therein). Hence, Theorem 1.1 and Theorem 1.2 imply the sufficiency part of Theorem A and Theorem B, respectively. However, due to the complexity of the symbol $\phi$, we cannot obtain the necessities of the range of $q$ in Theorem 1.1 and Theorem 1.2.

This paper is organized as follows. The proofs of Theorem 1.1 and Theorem 1.2 are given in Sect. 2 and Sect. 3, respectively. To prove Theorem 1.1 and Theorem 1.2, we next need the following important lemmas, which play a key role in proving Theorem 1.1 and Theorem 1.2, respectively. The proof of Lemma 1.4 is given in Sect. 4.

Lemma 1.3 ([26]) Assume that $\phi$ satisfies (H1)-(H3) with $m_{1}>1, m_{2}>1 . \frac{1}{2} \leq s<1$ and $\mu \in C_{0}^{\infty}(\mathbb{R})$. Then

$$
\left.\left|\int_{\mathbb{R}} e^{i x \xi+i t \phi(|\xi|)}\right| \xi\right|^{-s} \mu\left(\frac{\xi}{N}\right) d \xi \mid \leq C \frac{1}{|x|^{1-s}}
$$

for $x \in \mathbb{R} \backslash\{0\}, t \in \mathbb{R}$, and $N=1,2,3, \ldots$. Here the constant $C$ may depend on $s$ and $m_{1}, m_{2}$, and $\mu$ but not on $x, t$, or $N$.

Remark 1.2 The proof of Lemma 1.3 is similar to that of Lemma 2.1 in [10].

Lemma 1.4 Assume that $\phi$ satisfies (H1)-(H3) with $m_{1}>1, m_{2}>1 . \frac{1}{2} \leq \alpha \leq \frac{m_{2}}{2},-1<d<$ 1 , and $\mu \in C_{0}^{\infty}(\mathbb{R})$. Then

$$
\left|\int_{\mathbb{R}} \frac{e^{i(d \phi(\mid \xi)-x \xi)}}{\left(1+\xi^{2}\right)^{\frac{\alpha}{2}}} \mu\left(\frac{\xi}{N}\right) d \xi\right| \leq C \frac{1}{|x|^{\beta}}
$$

for $x \in \mathbb{R} \backslash\{0\}$ and $N=1,2,3, \ldots$, where $\beta=\frac{\alpha+\frac{m_{2}}{2}-1}{m_{2}-1}$. Here the constant $C$ may depend on $\alpha$ and $m_{1}, m_{2}$, and $\mu$ but not on $x, d$, and $N$.

Remark 1.3 Applying the result of Lemma 1.3, the proof of Lemma 1.4 is similar to that of Lemma 2.2 in [32]. The proof of Lemma 1.4 will be given in Sect. 4.

\section{The proof of Theorem 1.1}

Assume that $n \geq 2, \phi_{i}(i=1,2,3, \ldots, n)$ satisfies (H1)-(H3). For $i=1,2,3, \ldots, n$, let $t_{i}(x)$ be a measurable function on $\mathbb{R}^{n}$ with $t_{i}(x) \in \mathbb{R}$. Denote $t(x)=\left(t_{1}(x), t_{2}(x), \ldots, t_{n}(x)\right)$, we set

$$
S_{t(x), \Phi} f(x)=(2 \pi)^{-n} \int_{\mathbb{R}^{n}} e^{i x \cdot \xi} e^{i\left(t_{1}(x) \phi_{1}\left(\left|\xi_{1}\right|\right)+t_{2}(x) \phi_{2}\left(\left|\xi_{2}\right|\right)+\cdots+t_{n}(x) \phi_{n}\left(\left|\xi_{n}\right|\right)\right)} \hat{f}(\xi) d \xi,
$$




$$
x \in \mathbb{R}^{n}, f \in \mathcal{S}\left(\mathbb{R}^{n}\right)
$$

For $4 \leq q<\infty$ and $s=n\left(\frac{1}{2}-\frac{1}{q}\right)$, that is, $\frac{n}{4} \leq s<\frac{n}{2}$ and $q=\frac{2 n}{n-2 s}$. By linearizing the maximal operator (see [30]) to prove the global estimate (1.8) holds, it suffices to show that

$$
\left\|S_{t(x), \Phi} f\right\|_{L^{q\left(\mathbb{R}^{n}\right)}} \leq C\|f\|_{\dot{H}^{s}}=C\left(\int_{\mathbb{R}^{n}}|\xi|^{2 s}|\hat{f}(\xi)|^{2} d \xi\right)^{1 / 2}
$$

To prove (2.1) it suffices to prove that

$$
\left\|S_{t(x), \Phi} f\right\|_{L^{q}\left(\mathbb{R}^{n}\right)} \leq C\left(\left.\int_{\mathbb{R}^{n}}\left|\xi_{1}\right|^{\frac{2 s}{n}}\left|\xi_{2}\right|^{\frac{2 s}{n}}|\cdots| \xi_{n}\right|^{\frac{2 s}{n}}|\hat{f}(\xi)|^{2} d \xi\right)^{1 / 2}
$$

Let $g(\xi)=\left|\xi_{1}\right|^{\frac{s}{n}}\left|\xi_{2}\right|^{\frac{s}{n}} \cdots\left|\xi_{n}\right|^{\frac{s}{n}} \hat{f}(\xi)$, then we have

$$
\begin{aligned}
S_{t(x), \Phi} f(x) & =\int_{\mathbb{R}^{n}} e^{i x \cdot \xi} e^{i\left(t_{1}(x) \phi_{1}\left(\left|\xi_{1}\right|\right)+t_{2}(x) \phi_{2}\left(\left|\xi_{2}\right|\right)+\cdots+t_{n}(x) \phi_{n}\left(\left|\xi_{n}\right|\right)\right)}\left|\xi_{1}\right|^{-\frac{s}{n}}\left|\xi_{2}\right|^{-\frac{s}{n}} \cdots\left|\xi_{n}\right|^{-\frac{s}{n}} g(\xi) d \xi \\
& =R_{\Phi} g(x)
\end{aligned}
$$

where

$$
R_{\Phi} g(x)=\int_{\mathbb{R}^{n}} e^{i x \cdot \xi} e^{i\left(t_{1}(x) \phi_{1}\left(\left|\xi_{1}\right|\right)+t_{2}(x) \phi_{2}\left(\left|\xi_{2}\right|\right)+\cdots+t_{n}(x) \phi_{n}\left(\left|\xi_{n}\right|\right)\right)}\left|\xi_{1}\right|^{-\frac{s}{n}}\left|\xi_{2}\right|^{-\frac{s}{n}} \cdots\left|\xi_{n}\right|^{-\frac{s}{n}} g(\xi) d \xi
$$

To prove (2.2) it suffices to prove that

$$
\left\|R_{\Phi} g\right\|_{L^{q}\left(\mathbb{R}^{n}\right)} \leq C\|g\|_{L^{2}\left(\mathbb{R}^{n}\right)}
$$

for $g$ continuous and rapidly decreasing at infinity. We take a real-valued function $\rho \in$ $C_{0}^{\infty}\left(\mathbb{R}^{n}\right)$ such that $\rho(x)=1$ if $|x| \leq 1$ and $\rho(x)=0$ if $|x| \geq 2$. And we choose a real-valued function $\psi \in C_{0}^{\infty}(\mathbb{R})$ such that $\psi(x)=1$ if $|x| \leq 1$ and $\psi(x)=0$ if $|x| \geq 2$, and set $\sigma(\xi)=$ $\psi\left(\xi_{1}\right) \psi\left(\xi_{2}\right) \cdots \psi\left(\xi_{n}\right)$. For $\xi \in \mathbb{R}^{n}$ and $N=1,2,3, \ldots$, we set $\rho_{N}(x)=\rho\left(\frac{x}{N}\right)$ and $\sigma_{N}(\xi)=\sigma\left(\frac{\xi}{N}\right)$. For $x \in \mathbb{R}^{n}, g \in L^{2}\left(\mathbb{R}^{n}\right)$, and for $N=1,2,3, \ldots$, we define

$$
\begin{aligned}
R_{N, \Phi} g(x)= & \rho_{N}(x) \int_{\mathbb{R}^{n}} e^{i x \cdot \xi} e^{i\left(t_{1}(x) \phi_{1}\left(\left|\xi_{1}\right|\right)+t_{2}(x) \phi_{2}\left(\left|\xi_{2}\right|\right)+\cdots+t_{n}(x) \phi_{n}\left(\left|\xi_{n}\right|\right)\right)}\left|\xi_{1}\right|^{-\frac{s}{n}}\left|\xi_{2}\right|^{-\frac{s}{n}} \ldots \\
& \times\left|\xi_{n}\right|^{-\frac{s}{n}} \sigma_{N}(\xi) g(\xi) d \xi .
\end{aligned}
$$

The adjoint of $R_{N, \Phi}$ is given by

$$
\begin{aligned}
R_{N, \Phi}^{\prime} h(\xi)= & \sigma_{N}(\xi)\left|\xi_{1}\right|^{-\frac{s}{n}}\left|\xi_{2}\right|^{-\frac{s}{n}} \ldots \\
& \times\left|\xi_{n}\right|^{-\frac{s}{n}} \int_{\mathbb{R}^{2}} e^{-i x \cdot \xi} e^{-i\left(t_{1}(x) \phi_{1}\left(\left|\xi_{1}\right|\right)+t_{2}(x) \phi_{2}\left(\left|\xi_{2}\right|\right)+\cdots+t_{n}(x) \phi_{n}\left(\left|\xi_{n}\right|\right)\right)} \rho_{N}(x) h(x) d x,
\end{aligned}
$$

where $\xi \in \mathbb{R}^{n}$ and $h \in L^{2}\left(\mathbb{R}^{n}\right)$. To prove (2.4) it is sufficient to prove that

$$
\left\|R_{N, \Phi} g\right\|_{L^{q}\left(\mathbb{R}^{n}\right)} \leq C\|g\|_{L^{2}\left(\mathbb{R}^{n}\right)} .
$$


By duality, to prove (2.5) it suffices to show that

$$
\left\|R_{N, \Phi}^{\prime} h\right\|_{L^{2}\left(\mathbb{R}^{n}\right)} \leq C\|h\|_{L^{q^{\prime}}\left(\mathbb{R}^{n}\right)}
$$

where $\frac{1}{q}+\frac{1}{q^{\prime}}=1$. Thus, we have

$$
\left\|R_{N, \Phi}^{\prime} h\right\|_{L^{2}\left(\mathbb{R}^{n}\right)}^{2}=\int\left|R_{N, \Phi}^{\prime} h(\xi)\right|^{2} d \xi=\int_{\mathbb{R}^{n}} \int_{\mathbb{R}^{n}} K_{N}(x, y) \rho_{N}(x) \rho_{N}(y) h(x) \overline{h(y)} d x d y,
$$

where

$$
K_{N}(x, y)=K_{N}^{1}(x, y) K_{N}^{2}(x, y) \cdots K_{N}^{n}(x, y)
$$

and

$$
K_{N}^{i}(x, y)=\int_{\mathbb{R}}\left|\xi_{i}\right|^{-\frac{2 s}{n}} e^{i\left(y_{i}-x_{i}\right) \xi_{i}} e^{i\left(t_{i}(y)-t_{i}(x)\right) \phi\left(\left|\xi_{i}\right|\right)} \psi_{N}\left(\xi_{i}\right)^{2} d \xi_{i},
$$

where $i=1,2, \ldots, n$ and $N=1,2, \ldots$ Since $\frac{n}{4} \leq s<\frac{n}{2}$, we have $\frac{1}{2} \leq \frac{2 s}{n}<1$. Therefore, by Lemma $1.3,(2.9)$, and (2.8), we obtain

$$
\left|K_{N}(x, y)\right| \leq C \frac{1}{\left|x_{1}-y_{1}\right|^{1-\frac{2 s}{n}}} \frac{1}{\left|x_{2}-y_{2}\right|^{1-\frac{2 s}{n}}} \cdots \frac{1}{\left|x_{n}-y_{n}\right|^{1-\frac{2 s}{n}}} .
$$

We define

$$
P_{i} f\left(x_{1}, x_{2}, \ldots, x_{n}\right)=\int_{\mathbb{R}} \frac{1}{\left|x_{i}-y_{i}\right|^{1-\frac{2 s}{n}}} f\left(x_{1}, \ldots, x_{i-1}, y_{i}, x_{i+1}, \ldots, x_{n}\right) d y_{i},
$$

$i=1,2, \ldots, n$. Thus, by (2.7) and (2.10), we obtain

$$
\begin{aligned}
\int\left|R_{N, \Phi}^{\prime} h(\xi)\right|^{2} d \xi \\
\leq C \int_{\mathbb{R}^{n}} \int_{\mathbb{R}^{n}} \frac{1}{\left|x_{1}-y_{1}\right|^{1-\frac{2 s}{n}}} \frac{1}{\left|x_{2}-y_{2}\right|^{1-\frac{2 s}{n}}} \cdots \frac{1}{\left|x_{n}-y_{n}\right|^{1-\frac{2 s}{n}}}|h(x)||h(y)| d x d y \\
=C \int_{\mathbb{R}^{n}}\left(\int_{\mathbb{R}} \int_{\mathbb{R}} \int_{\mathbb{R}} \int_{\mathbb{R}} \frac{1}{\left|x_{n}-y_{n}\right|^{1-\frac{2 s}{n}}} \frac{1}{\left|x_{n-1}-y_{n-1}\right|^{1-\frac{2 s}{n}}} \cdots \frac{1}{\left|x_{3}-y_{3}\right|^{1-\frac{2 s}{n}}}\right. \\
\left.\quad \times \frac{1}{\left|x_{2}-y_{2}\right|^{1-\frac{2 s}{n}}}\left(\int \frac{1}{\left|x_{1}-y_{1}\right|^{1-\frac{2 s}{n}}}\left|h\left(y_{1}, y_{2}, \ldots, y_{n}\right)\right| d y_{1}\right) d y_{2} d y_{3} \cdots d y_{n}\right)|h(x)| d x \\
=C \int_{\mathbb{R}^{n}} P_{n} P_{n-1} \cdots P_{2} P_{1}|h|(x)|h(x)| d x .
\end{aligned}
$$

Invoking Hölder's inequality, we get

$$
\int\left|R_{N, \Phi}^{\prime} h(\xi)\right|^{2} d \xi \leq C\left\|P_{n} P_{n-1} \cdots P_{2} P_{1}|h|\right\|_{L^{q\left(\mathbb{R}^{n}\right)}}\|h\|_{L^{q^{\prime}\left(\mathbb{R}^{n}\right)}}
$$


Since $q=\frac{2 n}{n-2 s}$, it follows that $q^{\prime}=\frac{2 n}{n+2 s}$ and the fact $\frac{1}{q}=\frac{1}{q^{\prime}}-\frac{2 s}{n}$. Denote by $I_{\sigma}$ the Riesz potential of order $\sigma$, which is defined by

$$
I_{\sigma}(f)(u)=\int_{\mathbb{R}} \frac{f(v)}{|u-v|^{1-\sigma}} d v .
$$

Applying the fact $I_{s}$ is bounded from $L^{q^{\prime}}(\mathbb{R})$ to $L^{q}(\mathbb{R})$, we have

$$
\left(\int_{\mathbb{R}}\left|P_{j} h(x)\right|^{q} d x_{j}\right)^{1 / q} \leq C\left(\int_{\mathbb{R}}|h(x)|^{q^{\prime}} d x_{j}\right)^{1 / q^{\prime}}
$$

where $j=1,2, \ldots, n$. By (2.13) and Minkowski's inequality, we have

$$
\left\|P_{n} P_{n-1} \cdots P_{2} P_{1}|h|\right\|_{L^{q\left(\mathbb{R}^{n}\right)}} \leq C\|h\|_{L^{q^{\prime}}\left(\mathbb{R}^{n}\right)} .
$$

Therefore, (2.6) follows from (2.12) and (2.14). Now we complete the proof of Theorem 1.1.

\section{The proof of Theorem 1.2}

Assume that $n \geq 2, \phi_{i}(i=1,2,3, \ldots, n)$ satisfies (H1)-(H3) with $m_{i, 1}>1, m_{i, 2}>1$. For every $m=\left(m_{1,2}, m_{2,2}, \ldots, m_{n, 2}\right)$ and $2<q<4$, we will prove that inequality (1.9) holds if $s=\frac{n}{2}-$ $\frac{|m|}{4}+\frac{|m|}{q}-\frac{n}{q}$, where $|m|=m_{1,2}+m_{2,2}+\cdots+m_{n, 2}$. For $i=1,2,3, \ldots, n$, let $t_{i}(x)$ be a measurable function on $\mathbb{R}^{n}$ with $0<t_{i}(x)<1$. Denote $t(x)=\left(t_{1}(x), t_{2}(x), \ldots, t_{n}(x)\right)$, we set

$$
S_{t(x), \Phi} f(x)=\int_{\mathbb{R}^{n}} e^{i x \cdot \xi} e^{i\left(t_{1}(x) \phi_{1}\left(\left|\xi_{1}\right|\right)+t_{2}(x) \phi_{2}\left(\left|\xi_{2}\right|\right)+\cdots+t_{n}(x) \phi_{n}\left(\left|\xi_{n}\right|\right)\right)} \hat{f}(\xi) d \xi, \quad x \in \mathbb{R}^{n}, f \in \mathcal{S}\left(\mathbb{R}^{n}\right) .
$$

By linearizing the maximal operator, to prove the global estimate (1.9) it suffices to show that

$$
\left\|S_{t(x), \Phi} f\right\|_{L^{q}\left(\mathbb{R}^{n}\right)} \leq C\|f\|_{H^{s}}=C\left(\int_{\mathbb{R}^{n}}\left(1+|\xi|^{2}\right)^{s}|\hat{f}(\xi)|^{2} d \xi\right)^{1 / 2}
$$

Since $s=\frac{n}{2}-\frac{|m|}{4}+\frac{|m|}{q}-\frac{n}{q}=n \frac{1}{2}-\frac{m_{1,2}+m_{2,2}+\cdots+m_{n, 2}}{4}+\frac{m_{1,2}+m_{2,2}+\cdots+m_{n, 2}}{q}-n \frac{1}{q}=: s_{1}+s_{2}+\cdots+s_{n}$, where $s_{i}=\frac{1}{2}-\frac{m_{i, 2}}{4}+\frac{m_{i, 2}}{q}-\frac{1}{q}, i=1,2, \ldots, n$. Therefore, to prove (3.1) it suffices to prove that

$$
\left\|S_{t(x), \Phi} f\right\|_{L^{q}\left(\mathbb{R}^{n}\right)} \leq C\left(\int_{\mathbb{R}^{2}}\left(1+\left|\xi_{1}\right|^{2}\right)^{s_{1}}\left(1+\left|\xi_{2}\right|^{2}\right)^{s_{2}}|\cdots|\left(1+\left|\xi_{n}\right|^{2}\right)^{s_{n}}|\hat{f}(\xi)|^{2} d \xi\right)^{1 / 2}
$$

Let $g(\xi)=\left(1+\left|\xi_{1}\right|^{2}\right)^{\frac{s_{1}}{2}}\left(1+\left|\xi_{2}\right|^{2}\right)^{\frac{s_{2}}{2}} \cdots\left(1+\left|\xi_{n}\right|^{2}\right)^{\frac{s_{n}}{2}} \hat{f}(\xi)$, then we have

$$
S_{t(x), \Phi} f(x)=R_{\Phi} g(x),
$$

where

$$
\begin{aligned}
R_{\Phi} g(x)= & \int_{\mathbb{R}^{n}} e^{i x \cdot \xi} e^{i\left(t_{1}(x) \phi_{1}\left(\left|\xi_{1}\right|\right)+t_{2}(x) \phi_{2}\left(\left|\xi_{2}\right|\right)+\cdots+t_{n}(x) \phi_{n}\left(\left|\xi_{n}\right|\right)\right)}\left(1+\left|\xi_{1}\right|^{2}\right)^{-\frac{s_{1}}{2}}\left(1+\left|\xi_{2}\right|^{2}\right)^{-\frac{s_{2}}{2}} \cdots \\
& \times\left(1+\left|\xi_{n}\right|^{2}\right)^{-\frac{s_{n}}{2}} g(\xi) d \xi .
\end{aligned}
$$


By (3.3), to prove (3.2) it is sufficient to show that

$$
\left\|R_{\Phi} g\right\|_{L^{q}\left(\mathbb{R}^{n}\right)} \leq C\|g\|_{L^{2}\left(\mathbb{R}^{n}\right)}
$$

for $g$ continuous and rapidly decreasing at infinity. We take a real-valued function $\rho \in$ $C_{0}^{\infty}\left(\mathbb{R}^{n}\right)$ such that $\rho(x)=1$ if $|x| \leq 1$ and $\rho(x)=0$ if $|x| \geq 2$. And we choose a real-valued function $\psi \in C_{0}^{\infty}(\mathbb{R})$ such that $\psi(x)=1$ if $|x| \leq 1$ and $\psi(x)=0$ if $|x| \geq 2$, and $\operatorname{set} \sigma(\xi)=$ $\psi\left(\xi_{1}\right) \psi\left(\xi_{2}\right) \cdots \psi\left(\xi_{n}\right)$ for $\xi \in \mathbb{R}^{n}$. For $N=1,2,3, \ldots$, we set $\rho_{N}(x)=\rho\left(\frac{x}{N}\right)$ and $\sigma_{N}(\xi)=\sigma\left(\frac{\xi}{N}\right)$. For $x \in \mathbb{R}^{n}, g \in L^{2}\left(\mathbb{R}^{n}\right)$, and $N=1,2,3, \ldots$, we define

$$
\begin{aligned}
R_{N, \Phi} g(x)= & \rho_{N}(x) \int_{\mathbb{R}^{n}} e^{i x \cdot \xi} e^{i\left(t_{1}(x) \phi_{1}\left(\left|\xi_{1}\right|\right)+t_{2}(x) \phi_{2}\left(\left|\xi_{2}\right|\right)+\cdots+t_{n}(x) \phi_{n}\left(\left|\xi_{n}\right|\right)\right)}\left(1+\left|\xi_{1}\right|^{2}\right)^{-\frac{s_{1}}{2}}\left(1+\left|\xi_{2}\right|^{2}\right)^{-\frac{s_{2}}{2}} \\
& \times \cdots \times\left(1+\left|\xi_{n}\right|^{2}\right)^{-\frac{s_{n}}{2}} \sigma_{N}(\xi) g(\xi) d \xi .
\end{aligned}
$$

The adjoint of $R_{N, \Phi}$ is given by

$$
\begin{aligned}
R_{N, \Phi}^{\prime} h(\xi)= & \sigma_{N}(\xi)\left(1+\left|\xi_{1}\right|^{2}\right)^{-\frac{s_{1}}{2}}\left(1+\left|\xi_{2}\right|^{2}\right)^{-\frac{s_{2}}{2}} \cdots\left(1+\left|\xi_{n}\right|^{2}\right)^{-\frac{s_{n}}{2}} \int_{\mathbb{R}^{2}} e^{-i x \cdot \xi} e^{-i t_{1}(x) \phi_{1}\left(\left|\xi_{1}\right|\right)} \\
& \times e^{-i\left(t_{2}(x) \phi_{2}\left(\left|\xi_{2}\right|\right)+\cdots+t_{n}(x) \phi_{n}\left(\left|\xi_{n}\right|\right)\right)} \rho_{N}(x) h(x) d x
\end{aligned}
$$

where $\xi \in \mathbb{R}^{n}$ and $h \in L^{2}\left(\mathbb{R}^{n}\right)$. To prove (3.4) it suffices to prove that

$$
\left\|R_{N, \Phi} g\right\|_{L^{q}\left(\mathbb{R}^{n}\right)} \leq C\|g\|_{L^{2}\left(\mathbb{R}^{n}\right)} .
$$

By duality, to prove (3.5) it is sufficient to show that

$$
\left\|R_{N, \Omega}^{\prime} h\right\|_{L^{2}\left(\mathbb{R}^{n}\right)} \leq C\|h\|_{L^{q^{\prime}\left(\mathbb{R}^{n}\right)}},
$$

where $\frac{1}{q}+\frac{1}{q^{\prime}}=1$. Thus, we have

$$
\left\|R_{N, \Phi}^{\prime} h\right\|_{L^{2}\left(\mathbb{R}^{n}\right)}^{2}=\int\left|R_{N, \Phi}^{\prime} h(\xi)\right|^{2} d \xi=\int_{\mathbb{R}^{n}} \int_{\mathbb{R}^{n}} K_{N}(x, y) \rho_{N}(x) \rho_{N}(y) h(x) \overline{h(y)} d x d y,
$$

where

$$
K_{N}(x, y)=K_{N}^{1}(x, y) K_{N}^{2}(x, y) \cdots K_{N}^{n}(x, y)
$$

and

$$
K_{N}^{i}(x, y)=\int_{\mathbb{R}}\left(1+\xi_{i}^{2}\right)^{-s_{i}} e^{i\left(y_{i}-x_{i}\right) \xi_{i}} e^{i\left(t_{i}(y)-t_{i}(x)\right) \phi\left(\left|\xi_{i}\right|\right)} \psi_{N}\left(\xi_{i}\right)^{2} d \xi_{i},
$$

where $i=1,2, \ldots, n$ and $N=1,2, \ldots$ Denote $\alpha_{i}=2 s_{i}$, since $s_{i}=\frac{1}{2}-\frac{m_{i, 2}}{4}+\frac{m_{i, 2}}{q}-\frac{1}{q}, i=$ $1,2, \ldots, n$, and $2<q<4$, it follows that $\frac{1}{2}<\alpha_{i}<\frac{m_{i, 2}}{2}, i=1,2, \ldots, n$. Therefore, by (3.9) and Lemma 1.4, we obtain

$$
\left|K_{N}^{i}(x, y)\right| \leq C \frac{1}{\left|x_{i}-y_{i}\right|^{\beta_{i}}}
$$


where $\beta_{i}=\frac{\alpha_{i}+\frac{m_{i, 2}}{2}-1}{m_{i, 2}-1}$. Denote $\sigma_{i}=1-\beta_{i}$, we define

$$
P_{i} f\left(x_{1}, x_{2}, \ldots, x_{n}\right)=\int_{\mathbb{R}} \frac{1}{\left|x_{i}-y_{i}\right|^{1-\sigma_{i}}} f\left(x_{1}, \ldots, x_{i-1}, y_{i}, x_{i+1}, \ldots, x_{n}\right) d y_{i},
$$

$i=1,2, \ldots, n$. Thus, by (3.7), (3.8), and (3.10), we obtain

$$
\begin{aligned}
& \int\left|R_{N, \Phi}^{\prime} h(\xi)\right|^{2} d \xi \\
& \leq C \int_{\mathbb{R}^{n}} \int_{\mathbb{R}^{n}} \frac{1}{\left|x_{1}-y_{1}\right|^{1-\sigma_{1}}} \frac{1}{\left|x_{2}-y_{2}\right|^{1-\sigma_{2}}} \cdots \frac{1}{\left|x_{n}-y_{n}\right|^{1-\sigma_{n}}}|h(x)||h(y)| d x d y \\
& =C \int_{\mathbb{R}^{n}}\left(\int_{\mathbb{R}} \int_{\mathbb{R}} \int_{\mathbb{R}} \int_{\mathbb{R}} \frac{1}{\left|x_{n}-y_{n}\right|^{1-\sigma_{n}}} \frac{1}{\left|x_{n-1}-y_{n-1}\right|^{1-\sigma_{n-1}}} \cdots \frac{1}{\left|x_{3}-y_{3}\right|^{1-\sigma_{3}}}\right. \\
& \left.\quad \times \frac{1}{\left|x_{2}-y_{2}\right|^{1-\sigma_{2}}}\left(\int \frac{1}{\left|x_{1}-y_{1}\right|^{1-\sigma_{1}}}\left|h\left(y_{1}, y_{2}, \ldots, y_{n}\right)\right| d y_{1}\right) d y_{2} d y_{3} \cdots d y_{n}\right)|h(x)| d x \\
& =C \int_{\mathbb{R}^{n}} P_{n} P_{n-1} \cdots P_{2} P_{1}|h|(x)|h(x)| d x .
\end{aligned}
$$

Invoking Hölder's inequality, we get

$$
\int\left|R_{N, \Phi}^{\prime} h(\xi)\right|^{2} d \xi \leq C\left\|P_{n} P_{n-1} \cdots P_{2} P_{1}|h|\right\|_{L^{q}\left(\mathbb{R}^{n}\right)}\|h\|_{L^{q^{\prime}}\left(\mathbb{R}^{n}\right)} .
$$

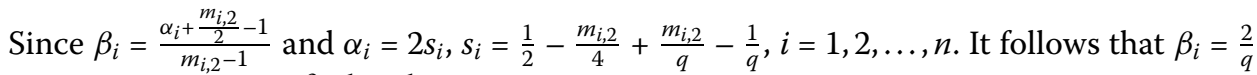
and $\sigma_{i}=1-\beta_{i}=1-\frac{2}{q}, \frac{1}{q}=\frac{1}{q^{\prime}}-\sigma_{i}$. Thus, estimate (3.6) follows from (3.12) and estimate (2.14) in the proof of Theorem 1.1. Now we complete the proof of Theorem 1.2.

\section{The proof of Lemma 1.4}

To prove Lemma 1.4, we need to present the following lemma.

Lemma 4.1 (see [33], pp. 309-312) Assume that $a<b$ and set $I=[a, b]$. Let $F \in C^{\infty}(I)$ be real-valued and assume that $\psi \in C^{\infty}(I)$.

(i) Assume that $\left|F^{\prime}(x)\right| \geq \lambda>0$ for $x \in I$ and that $F^{\prime}$ is monotonic on I. Then

$$
\left|\int_{a}^{b} e^{i F(x)} \psi(x) d x\right| \leq C \frac{1}{\lambda}\left\{|\psi(b)|+\int_{a}^{b}\left|\psi^{\prime}(x)\right| d x\right\},
$$

where $C$ does not depend on $F, \psi$, or $I$.

(ii) Assume that $\left|F^{\prime \prime}(x)\right| \geq \lambda>0$ for $x \in I$. Then

$$
\left|\int_{a}^{b} e^{i F(x)} \psi(x) d x\right| \leq C \frac{1}{\lambda^{1 / 2}}\left\{|\psi(b)|+\int_{a}^{b}\left|\psi^{\prime}(x)\right| d x\right\},
$$

where $C$ does not depend on $F, \psi$, or $I$.

Proof of Lemma 1.4 By conditions (H1) and (H2), there exist positive constants $C_{i}(i=$ $1,2, \ldots, 6)$ so that for $r \geq 1$ and $m_{2}>1$ such that

$$
C_{1} r^{m_{2}-1} \leq\left|\phi^{\prime}(r)\right| \leq C_{2} r^{m_{2}-1} \quad \text { and } \quad\left|\phi^{\prime \prime}(r)\right| \geq C_{3} r^{m_{2}-2}
$$


and for $0<r<1$ and $m_{1}>1$ such that

$$
C_{4} r^{m_{1}-1} \leq\left|\phi^{\prime}(r)\right| \leq C_{5} r^{m_{1}-1} \quad \text { and } \quad\left|\phi^{\prime \prime}(r)\right| \geq C_{6} r^{m_{1}-2} \text {. }
$$

Set

$$
J=\int_{\mathbb{R}} \frac{e^{i(d \phi(|\xi|)-x \xi)}}{\left(1+\xi^{2}\right)^{\frac{\alpha}{2}}} \mu\left(\frac{\xi}{N}\right) d \xi
$$

To prove Lemma 1.4, it suffices to show that there exists a constant $C$ such that for $x \in$ $\mathbb{R} \backslash\{0\}, \beta=\frac{\alpha+\frac{m_{2}}{2}-1}{m_{2}-1}$ and $N \in \mathbb{N}$,

$$
|J| \leq C \frac{1}{|x|^{\beta}}
$$

where $C$ depends only on $\alpha, m_{1}, m_{2}, C_{i}(i=1,2, \ldots, 6)$, and $\mu$.

Without loss of generality, we may assume $\xi, d>0$. Denote $\psi(\xi)=\left(1+\xi^{2}\right)^{-\frac{\alpha}{2}} \mu\left(\frac{\xi}{N}\right)$, then we have

$$
\max _{\xi \geq 0}|\psi(\xi)|+\int_{0}^{\infty}\left|\psi^{\prime}(\xi)\right| d \xi \leq C
$$

In fact, since $\mu \in C_{0}^{\infty}(\mathbb{R})$ and $\frac{1}{2} \leq \alpha \leq \frac{m_{2}}{2}$, we get

$$
\max _{\xi \geq 0}|\psi(\xi)| \leq C
$$

Noting that

$$
\psi^{\prime}(\xi)=-\alpha \xi\left(1+\xi^{2}\right)^{-\frac{\alpha}{2}-1} \mu\left(\frac{\xi}{N}\right)+\left(1+\xi^{2}\right)^{-\frac{\alpha}{2}} \frac{1}{N} \mu^{\prime}\left(\frac{\xi}{N}\right)
$$

we have

$$
\begin{aligned}
\int_{0}^{\infty}\left|\psi^{\prime}(\xi)\right| d \xi \leq & \alpha \int_{0}^{\infty} \xi\left(1+\xi^{2}\right)^{-\frac{\alpha}{2}-1}\left|\mu\left(\frac{\xi}{N}\right)\right| d \xi \\
& +\int_{0}^{\infty}\left(1+\xi^{2}\right)^{-\frac{\alpha}{2}} \frac{1}{N}\left|\mu^{\prime}\left(\frac{\xi}{N}\right)\right| d \xi \\
=: & G_{1}+G_{2} .
\end{aligned}
$$

Since $\mu \in C_{0}^{\infty}(\mathbb{R})$ and $\frac{1}{2} \leq \alpha \leq \frac{m_{2}}{2}$, we obtain

$$
G_{1} \leq C \int_{0}^{\infty} \xi\left(1+\xi^{2}\right)^{-\frac{\alpha}{2}-1} d \xi=C \int_{0}^{\infty}\left(1+\xi^{2}\right)^{-\frac{\alpha}{2}-1} d\left(1+\xi^{2}\right)=C
$$

and

$$
G_{2} \leq C \int_{0}^{\infty} \frac{1}{N}\left|\mu^{\prime}\left(\frac{\xi}{N}\right)\right| d \xi \leq C
$$


By (4.7), (4.8), and (4.9), we get

$$
\int_{0}^{\infty}\left|\psi^{\prime}(\xi)\right| d \xi \leq C
$$

Therefore, (4.4) follows from (4.5) and (4.10).

To estimate (4.3), we choose a positive constant $M$ such that $M=\max \left\{\left(\frac{1}{\delta}\right)^{m_{2}-1}, 2 C_{5}, 2\right\}$, where $\delta$ is a small positive constant such that $\delta^{m_{2}-1} C_{2} \leq \frac{1}{2}$. Below, we show (4.3) by dividing two cases $|x| \geq M$ and $|x|<M$.

Case (I): $|x| \geq M$. Let $F(\xi)=d \phi(\xi)-x \xi$, we have

$$
F^{\prime}(\xi)=d \phi^{\prime}(\xi)-x, \quad F^{\prime \prime}(\xi)=d \phi^{\prime \prime}(\xi)
$$

Denote $\rho=\left(\frac{|x|}{d}\right)^{\frac{1}{m_{2}-1}}$, then we have $\delta \rho \geq 1$. In fact, noting that $|x| \geq\left(\frac{1}{\delta}\right)^{m_{2}-1}, 0<d<1$, $m_{2}>1$, and $\frac{|x|}{d}>|x|$, it follows that $\delta \rho>\delta|x|^{\frac{1}{m_{2}-1}} \geq 1$. We choose a large positive constant $\lambda$ such that $\lambda \geq \max \left\{\left(\frac{2}{C_{1}}\right)^{\frac{1}{m_{2}-1}}, \delta\right\}$. Denote

$$
I_{1}=[0, \delta \rho], \quad I_{2}=[\delta \rho, \lambda \rho], \quad I_{3}=[\lambda \rho, \infty) .
$$

Thus, we obtain

$$
|J|=\left|\int_{0}^{\infty} e^{i F(\xi)} \psi(\xi) d \xi\right| \leq \sum_{j=1}^{3}\left|\int_{I_{j}} e^{i F(\xi)} \psi(\xi) d \xi\right|=: \sum_{j=1}^{3} J_{j} .
$$

Firstly, we estimate $J_{1}$. We will show that the following estimate holds:

$$
\left|F^{\prime}(\xi)\right| \geq \frac{|x|}{2}, \quad \xi \in[0, \delta \rho]
$$

Now we divide the verification of (4.12) into two cases according to the value of $\xi$.

Case (I-a): $\xi \in[0,1)$. Since $m_{1}>1$ and $0<d<1$, we have

$$
d\left|\phi^{\prime}(\xi)\right| \leq C_{5} d \xi^{m_{1}-1} \leq C_{5} \leq \frac{M}{2} \leq \frac{|x|}{2}
$$

By (4.13), if $\xi \in[0,1)$, we get

$$
\left|F^{\prime}(\xi)\right| \geq|x|-d\left|\phi^{\prime}(\xi)\right| \geq \frac{|x|}{2}
$$

Case (I-b): $\xi \in[1, \delta \rho]$. Since $m_{2}>1$, we have

$$
d\left|\phi^{\prime}(\xi)\right| \leq C_{2} d \xi^{m_{2}-1} \leq C_{2} d \delta^{m_{2}-1} \frac{|x|}{d} \leq C_{2} \delta^{m_{2}-1}|x| \leq \frac{|x|}{2} .
$$

By (4.15), we get

$$
\left|F^{\prime}(\xi)\right| \geq|x|-d\left|\phi^{\prime}(\xi)\right| \geq \frac{|x|}{2}
$$


Therefore (4.12) follows from (4.14) and (4.16). Since $\phi^{\prime}$ is monotonic on $\mathbb{R}^{+}$by condition (H3) and $d>0$, it follows that $F^{\prime}$ is monotonic on $\xi \in I_{1}$. Thus, by (i) of Lemma 4.1 and estimate (4.12), (4.4), we have

$$
\left|J_{1}\right| \leq C \frac{1}{|x|} \leq C \frac{1}{|x|^{\beta}}
$$

where we use $|x| \geq 2$ and the fact $\frac{1}{2} \leq \beta \leq 1$. Next we prove estimate $J_{3}$. Since $\xi \geq$ $\lambda\left(\frac{|x|}{d}\right)^{\frac{1}{m_{2}-1}}>1$ and $\lambda \geq\left(\frac{2}{C_{1}}\right)^{\frac{1}{m_{2}-1}}$,

$$
d\left|\phi^{\prime}(\xi)\right| \geq C_{1} d \xi^{m_{2}-1} \geq C_{1} d \lambda^{m_{2}-1} \frac{|x|}{d} \geq 2|x|
$$

it follows that

$$
\left|F^{\prime}(\xi)\right| \geq 2|x|-|x|=|x|, \quad \xi \in[\lambda \rho, \infty) .
$$

Thus, by (i) of Lemma 4.1 and estimate (4.18), (4.4), we have

$$
\left|J_{3}\right| \leq C \frac{1}{|x|} \leq C \frac{1}{|x|^{\beta}}
$$

where we use $|x| \geq 2$ and the fact $\frac{1}{2} \leq \beta \leq 1$. Now, we give estimate $J_{2}$. Since $\xi \in I_{2}$, we have $|\xi| \geq 1$. By (4.1), we obtain

$$
\left|F^{\prime \prime}(\xi)\right| \geq d\left|\phi^{\prime \prime}(\xi)\right| \geq C_{3} d \xi^{m_{2}-2} \geq C_{3} d\left(\frac{|x|}{d}\right)^{\frac{m_{2}-2}{m_{2}-1}}
$$

We first prove that the following estimate holds:

$$
\max _{I_{2}}|\psi|+\int_{I_{2}}\left|\psi^{\prime}\right| d \xi \leq C\left(\frac{|x|}{d}\right)^{-\frac{\alpha}{m_{2}-1}} .
$$

In fact, since $\mu \in C_{0}^{\infty}(\mathbb{R})$ and $\frac{1}{2} \leq \alpha \leq \frac{m_{2}}{2}$, we get

$$
\max _{\xi \in A_{2}}|\psi(\xi)| \leq C(\delta \rho)^{-\alpha}=C \delta^{-\alpha}(\rho)^{-\alpha}=C \delta^{-\alpha}\left(\frac{|x|}{d}\right)^{-\frac{\alpha}{m_{2}-1}} .
$$

By (4.6), we have

$$
\begin{aligned}
\int_{A_{2}}\left|\psi^{\prime}(\xi)\right| d \xi & \leq \alpha \int_{A_{2}} \xi\left(1+\xi^{2}\right)^{-\frac{\alpha}{2}-1}\left|\mu\left(\frac{\xi}{N}\right)\right| d \xi+\int_{A_{2}}\left(1+\xi^{2}\right)^{-\frac{\alpha}{2}} \frac{1}{N}\left|\mu^{\prime}\left(\frac{\xi}{N}\right)\right| d \xi \\
& =: L_{1}+L_{2} .
\end{aligned}
$$

Since $\mu \in C_{0}^{\infty}(\mathbb{R})$ and $\frac{1}{2} \leq \alpha \leq \frac{m_{2}}{2}$, we obtain

$$
L_{1} \leq C \int_{A_{2}} \xi\left(1+\xi^{2}\right)^{-\frac{\alpha}{2}-1} d \xi \leq C \int_{\delta \rho}^{\lambda \rho} \xi^{-\alpha-1} d \xi=C\left(\frac{|x|}{d}\right)^{-\frac{\alpha}{m_{2}-1}}
$$


and

$$
L_{2} \leq C(\delta \rho)^{-\alpha} \int_{A_{2}} \frac{1}{N}\left|\mu^{\prime}\left(\frac{\xi}{N}\right)\right| d \xi \leq C\left(\frac{|x|}{d}\right)^{-\frac{\alpha}{m_{2}-1}} .
$$

By (4.23), (4.24), and (4.25), we get

$$
\int_{0}^{\infty}\left|\psi^{\prime}(\xi)\right| d \xi \leq C\left(\frac{|x|}{d}\right)^{-\frac{\alpha}{m_{2}-1}}
$$

Therefore, (4.21) follows from (4.22) and (4.26). Thus, by (ii) of Lemma 4.1 and estimate (4.20), (4.21), we have

$$
\left|J_{2}\right| \leq d^{-\frac{1}{2}}\left(\frac{|x|}{d}\right)^{-\frac{m_{2}-2}{2\left(m_{2}-1\right)}}\left(\frac{|x|}{d}\right)^{-\frac{\alpha}{m_{2}-1}}=C \frac{d^{\frac{\alpha-\frac{1}{2}}{m_{2}-1}}}{|x|^{\frac{\alpha+\frac{m_{2}}{2}-1}{m_{2}-1}}} \leq C \frac{1}{|x|^{\beta}} .
$$

Here in the last inequality we use the fact $\frac{\alpha-\frac{1}{2}}{m_{2}-1} \geq 0$ and $0<d<1$. Therefore, for $|x| \geq M$, by estimates (4.11), (4.17), (4.19), and (4.27), it follows (4.3).

Case (II): $|x|<M$. Now we divide the verification of (4.3) into three cases according to the value of $\alpha$ for $|x|<M$.

Case (II-a): $\alpha>1$. Since $\mu \in C_{0}^{\infty}(\mathbb{R})$ and $\alpha>1$, we get

$$
|J|=\left|\int_{0}^{\infty} \frac{e^{i(d \phi(\xi \mid)-x \xi)}}{\left(1+\xi^{2}\right)^{\frac{\alpha}{2}}} \mu\left(\frac{\xi}{N}\right) d \xi\right| \leq C \int_{0}^{\infty} \frac{1}{\left(1+\xi^{2}\right)^{\frac{\alpha}{2}}} d \xi \leq C .
$$

Noting that $|x|<M$ and $\frac{1}{2} \leq \beta \leq 1$, by (4.28), we have

$$
|J| \leq C=C|x|^{\beta} \frac{1}{|x|^{\beta}} \leq C M^{\beta} \frac{1}{|x|^{\beta}}=C \frac{1}{|x|^{\beta}},
$$

which follows (4.3).

Case (II-b): $\frac{1}{2} \leq \alpha<1$. By the mean value theorem, when $\frac{1}{2} \leq \alpha<1$, we have

$$
0<\left(1+\xi^{2}\right)^{\frac{\alpha}{2}}-\xi^{\alpha}=\left(1+\xi^{2}\right)^{\frac{\alpha}{2}}-\left(\xi^{2}\right)^{\frac{\alpha}{2}} \leq \frac{\alpha}{2}\left(\xi^{2}\right)^{\frac{\alpha}{2}-1} \leq \xi^{\alpha-2} .
$$

By (4.29), we obtain

$$
\frac{1}{\xi^{\alpha}}-\frac{1}{\left(1+\xi^{2}\right)^{\frac{\alpha}{2}}}=O\left(\frac{1}{\xi^{\alpha+2}}\right), \quad \xi \rightarrow \infty
$$

Noting that $\frac{1}{2} \leq \alpha<1$, by (4.30), we have

$$
\int_{0}^{\infty}\left|\frac{1}{\xi^{\alpha}}-\frac{1}{\left(1+\xi^{2}\right)^{\frac{\alpha}{2}}}\right| d \xi \leq C
$$

and

$$
|J|=\left|\int_{0}^{\infty} \frac{e^{i(d \phi(\xi \mid)-x \xi)}}{\left(1+\xi^{2}\right)^{\frac{\alpha}{2}}} \mu\left(\frac{\xi}{N}\right) d \xi\right| \leq\left|\int_{0}^{\infty} e^{i(d \phi(\xi \mid)-x \xi)}\left(\frac{1}{\left(1+\xi^{2}\right)^{\frac{\alpha}{2}}}-\frac{1}{\xi^{\alpha}}\right) \mu\left(\frac{\xi}{N}\right) d \xi\right|
$$




$$
\begin{aligned}
& +\left|\int_{0}^{\infty} e^{i(d \phi(|\xi|)-x \xi)} \frac{1}{\xi^{\alpha}} \mu\left(\frac{\xi}{N}\right) d \xi\right| \\
= & : K_{1}+K_{2} .
\end{aligned}
$$

By (4.31), we have

$$
\left|K_{1}\right| \leq C=C|x|^{\beta} \frac{1}{|x|^{\beta}} \leq C M^{\beta} \frac{1}{|x|^{\beta}}=C \frac{1}{|x|^{\beta}} .
$$

By Lemma 1.3, we obtain

$$
\left|K_{2}\right| \leq C \frac{1}{|x|^{1-\alpha}} .
$$

Noting that $\frac{1}{|x|}>\frac{1}{M}$, and the fact $\beta \geq 1-\alpha$, it follows from $\frac{1}{2} \leq \alpha<1, \frac{1}{2} \leq \beta \leq 1$ that

$$
|x|^{1-\alpha}=|x|^{\beta}|x|^{1-\alpha-\beta}=|x|^{\beta}\left(\frac{1}{|x|}\right)^{\beta-(1-\alpha)} \geq C|x|^{\beta} .
$$

Therefore, by (4.33) and (4.34), we have

$$
\left|K_{2}\right| \leq C \frac{1}{|x|^{\beta}} .
$$

Hence, (4.3) holds from (4.32) and (4.35).

Case (II-c): $\alpha=1$. From the proof of Lemma 1.3, noting that $M \geq 2$, we may get

$$
|J| \leq C \log \left(\frac{1}{|x|}\right) \quad \text { if } 0<|x| \leq \frac{1}{2}
$$

and

$$
|J| \leq C \quad \text { if } \frac{1}{2}<|x|<M .
$$

By (4.36) and $\frac{1}{2} \leq \beta \leq 1$, for $0<|x| \leq \frac{1}{2}$, we have

$$
|J| \leq C \log \left(\frac{1}{|x|}\right) \leq C \frac{1}{|x|^{\beta}} .
$$

By (4.37), for $\frac{1}{2}<|x|<M$, we have

$$
|J| \leq C=C|x|^{\beta} \frac{1}{|x|^{\beta}} \leq C M^{\beta} \frac{1}{|x|^{\beta}}=C \frac{1}{|x|^{\beta}} .
$$

Thus, for $\alpha=1,|x|<M$, by (4.38) and (4.39), we get

$$
|J| \leq C \frac{1}{|x|^{\beta}},
$$

which is just estimate (4.3).

Summing up all the above estimates, we complete the proof of estimate (4.3) and finish the proof of Lemma 1.4. 


\title{
5 Conclusion
}

In this paper, by linearizing the maximal operator and duality methods, and applying the results of Lemma 1.3 and Lemma 1.4, we obtain the maximal global $L^{q}$ inequalities (1.8) and (1.9) for multiparameter oscillatory integral $S_{t, \Phi}$. These estimates are apparently good extensions to maximal global $L^{q}$ inequalities (1.6) and (1.7) for the multiparameter fractional Schrödinger equation in [32].

\author{
Acknowledgements \\ The authors would like to express their deep gratitude to the anonymous referees for their careful reading of the \\ manuscript and their fruitful comments and suggestions.
}

\section{Funding}

The work is supported by NSFC (No. 11661061, No. 11561062, No. 11761054), Inner Mongolia University scientific research projects (No. NJZZ16234, NJZY17289).

\section{Competing interests}

The authors declare that they have no competing interests.

\section{Authors' contributions}

YN participated in the design of the study and in the discussions of all results. YX participated in the discussions of all results. All authors read and approved the final manuscript.

\section{Publisher's Note}

Springer Nature remains neutral with regard to jurisdictional claims in published maps and institutional affiliations.

Received: 28 June 2018 Accepted: 13 December 2018 Published online: 20 December 2018

\section{References}

1. Bourgain, J.: On the Schrödinger maximal function in higher dimension. Proc. Steklov Inst. Math. 280, 46-60 (2013)

2. Bourgain, J.: A note on the Schrödinger maximal function. J. Anal. Math. 130, 393-396 (2016)

3. Carbery, A.: Radial Fourier multipliers and associated maximal functions, recent progress in Fourier analysis. North-Holl. Math. Stud. 111, 49-56 (1985)

4. Carleson, L.: Some analytical problems related to statistical mechanics. Lect. Notes Math. 779, 5-45 (1979)

5. Cho, Y., Lee, S.: Strichartz estimates in spherical coordinates. Indiana Univ. Math. J. 62, 991-1020 (2013)

6. Cho, Y., Lee, S., Ozawa, T.: On small amplitude solutions to the generalized Boussinesq equations. Discrete Contin. Dyn. Syst., Ser. A 17, 691-711 (2007)

7. Cowling, M.: Pointwise behavior of solutions to Schrödinger equations. In: Lecture Notes in Math., vol. 992, pp. 83-90. Springer, Berlin (1983)

8. Dahlberg, B., Kenig, C.: A note on the almost everywhere behaviour of solutions to the Schrödinger equation. Lect. Notes Math. 908, 205-209 (1982)

9. Demeter, C., Guo, S.: Schrödinger maximal function estimates the pseudoconformal transformation. arXiv:1608.07640

10. Ding, Y., Niu, Y.: Weighted maximal estimates along curve associated with dispersive equations. Anal. Appl. 15, 225-240 (2017)

11. Du, X., Guth, L., Li, X.: A sharp Schrödinger maximal estimate in $\mathbb{R}^{2}$. Ann. Math. 186, 607-640 (2017)

12. Du, X., Guth, L., Li, X., Zhang, R.: Pointwise convergence of Schrödinger solutions and multilinear related Strichartz estimate. arXiv:1803.01720

13. Du, X., Zhang, R.: Sharp $L^{2}$ estimate of Schrödinger maximal function in higher dimensions. arXiv:1805.02775v1

14. Fefferman, R.: Multiparameter Fourier analysis. In: Beijing Lectures in Harmonic Analysis. Ann. Math. Stud., vol. 112, pp. 47-130. Princeton Univ. Press, Princeton (1986)

15. Fefferman, R.: Harmonic analysis on product spaces. Ann. Math. 126, 109-130 (1987)

16. Fefferman, R.: Multiparameter Calderón-Zygmund theory. In: Harmonic Analysis and Partial Differential Equations, Chicago. Chicago Lectures Math., pp. 207-221. Univ. Chicago Press, Chicago (1999)

17. Fefferman, R., Stein, E.M.: Singular integrals on product spaces. Adv. Math. 45, 117-143 (1982)

18. Frölich, J., Lenzmann, E.: Mean-field limit of quantum Bose gases and nonlinear Hartree equation. Semin. Equ. Deriv. Partielles 19, 1-26 (2004)

19. Guo, Z., Wang, Y.: Improved Strichartz estimates for a class of dispersive equations in the radial case and their applications to nonlinear Schrödinger and wave equations. J. Anal. Math. 124, 1-38 (2014)

20. Kenig, C., Ponce, G., Vega, L.: Well-posedness of the initial value problem for the Korteweg-de Vries equation. J. Am. Math. Soc. 4, 323-347 (1991)

21. Kenig, C., Ruiz, A.: A strong type (2; 2) estimate for a maximal operator associated to the Schrödinger equations. Trans. Am. Math. Soc. 280, 239-246 (1983)

22. Krieger, J., Lenzmann, E., Raphaël, P.: Nondispersive solutions to the $L^{2}$-critical half-wave equation. Arch. Ration. Mech. Anal. 209, 61-129 (2013)

23. Laskin, N.: Fractional quantum mechanics. Phys. Rev. E 62, 3135-3145 (2002)

24. Lee, S.: On pointwise convergence of the solutions to Schrödinger equations in $\mathbb{R}^{2}$. Int. Math. Res. Not. 2006, Article ID 32597 (2006) 
25. Lucà, R., Rogers, K.: An improved necessary condition for the Schrödinger maximal estimate. arXiv:1506.05325

26. Niu, Y., Xue, Y.: Global maximal estimate to some oscillatory integrals. Bull. Korean Math. Soc. 55, 533-543 (2018)

27. Peng, L., Wang, B.: Decay estimates for a class of wave equations. J. Funct. Anal. 254, 1642-1660 (2008)

28. Rogers, K.M.: A local smoothing estimate for the Schrödinger equation. Adv. Math. 219, 2105-2122 (2008)

29. Rogers, K.M., Villarroya, P.: Global estimates for the Schrödinger maximal operator. Ann. Acad. Sci. Fenn., Math. 32, 425-435 (2007)

30. Sjölin, P.: Regularity of solutions to the Schrödinger equation. Duke Math. J. 55, 699-715 (1987)

31. Sjölin, P.: Maximal estimates for solutions to the nonelliptic Schrödinger equation. Bull. Lond. Math. Soc. 39, 404-412 (2007)

32. Sjölin, P., Soria, F.: Estimates for multiparameter maximal operators of Schrödinger type. J. Math. Anal. Appl. 411, 129-143 (2014)

33. Stein, E.M.: Oscillatory integrals in Fourier analysis. In: Beijing Lectures in Harmonic Analysis. Ann. Math. Stud., vol. 112 pp. 307-355. Princeton Univ. Press, Princeton (1986)

34. Tao, T.: A sharp bilinear restrictions estimate for paraboloids. Geom. Funct. Anal. 13, 1359-1384 (2003)

35. Vega, L.: Schrödinger equations: pointwise convergence to the initial data. Proc. Am. Math. Soc. 102, 874-878 (1988)

\section{Submit your manuscript to a SpringerOpen ${ }^{\circ}$ journal and benefit from:}

- Convenient online submission

- Rigorous peer review

- Open access: articles freely available online

- High visibility within the field

- Retaining the copyright to your article

Submit your next manuscript at $\boldsymbol{\Delta}$ springeropen.com 